\title{
COMPLICAÇÕES PÓS-CIRÚRGICAS EM PACIENTES INFECTADOS POR COVID-19: REVISÃO INTEGRATIVA
}

\section{POST-SURGICAL COMPLICATIONS IN PATIENTS INFECTED BY COVID-19: INTEGRATIVE REVIEW}

\author{
Aline Sousa Falcão ${ }^{1}$ * Fábio França Silva ${ }^{2}$
}

\section{RESUMO}

Objetivo: identificar as principais evidências científicas disponíveis relativas às principais complicações pós-operatórias em pacientes Pós-COVID-19. Método: revisão integrativa, baseado em estudos publicados online nas seguintes bases de dados: PubMed/MEDLINE, Biblioteca Virtual em Saúde (BVS), SciELO, LILACS, com a questão norteadora: "Quais as atuais evidências científicas disponíveis na literatura a respeito das complicações pós-cirurgia em pacientes após infecção por COVID-19?" com critérios de inclusão: estudos que abordavam complicações cirúrgicas em pacientes após infecção por COVID-19, publicados em português e inglês, no ano de 2020 a 202. A estratégia de busca seguiu a estratégia PICOS conforme pressupostos do PRISMA. Resultados: 7 artigos foram selecionados. As seguintes complicações pós-operatórias em pacientes com COVID-19 foram identificadas: complicações pulmonares, complicações infecciosas (não pulmonares), lesão renal aguda, complicações tromboembólicas, choque séptico e aumento da mortalidade. Conclusão: A cirurgia pode tem um impacto negativo em pacientes COVID-19, mesmo os que são assintomáticos, elevando a mortalidade e a necessidade potencial de internação pós-operatória em Unidade de Terapia Intensiva (UTI), portanto, todos os procedimentos não urgentes devem ser adiados até que o paciente tenha cumprido com os critérios de isolamento e cuidados de transmissão e a Covid-19 tenha entrado na fase de recuperação.

Palavras-chave: COVID-19; Infecções por Coronavírus; Procedimento Cirúrgico; Intervenções Cirúrgicas; Complicações Pós-Operatórias.

\begin{abstract}
Objective: identify the main available scientific evidence regarding the main postoperative complications in Post-COVID-19 patients. Method: integrative review, based on studies published online in the following databases: PubMed/MEDLINE, Virtual Health Library (BVS), SciELO, LILACS, with the guiding question: "What is the current scientific evidence available in the literature regarding complications post-surgery in patients after COVID-19 infection?" with inclusion criteria: studies that addressed surgical complications in patients after infection by COVID-19, published in Portuguese and English, in the year 2020 to 202. The search strategy followed the PICOS strategy according to PRISMA assumptions. Results: 7 articles were selected. The following postoperative complications in patients with COVID-19 were identified: pulmonary complications, infectious (non-pulmonary) complications, acute kidney injury, thromboembolic complications, septic shock, and increased mortality. Conclusion: Surgery can have a negative impact on COVID-19 patients, even those who are asymptomatic, increasing mortality and the potential need for postoperative hospitalization in the Intensive Care Unit (ICU), therefore, all nonurgent procedures should be delayed until the patient has met criteria for isolation and transmission care and Covid-19 has entered the recovery phase.
\end{abstract}

Keywords: COVID-19; Coronavirus Infections; Surgical Procedure; Surgical Interventions; Postoperative Complications.

\footnotetext{
${ }^{1}$ Hospital Universitário da Universidade Federal do Maranhão (HU-UFMA), São Luís, Brasil. ORCID: https://orcid.org/0000-00031769-0012

${ }^{2}$ Hospital Universitário da Universidade Federal do Maranhão (HU-UFMA), São Luís, Brasil, ORCID: https://orcid.org/0000-0001$8700-1783$
} 
INTRODUÇÃO

A COVID-19, infecção causada pelo coronavírus SARS-CoV-2, foi identificado inicialmente em dezembro de 2019, em Wuhan, na China, com a ocorrência de casos de pneumonia em decorrência de um agente infeccioso, até então desconhecido. Posteriormente, foi identificado o alto poder de contágio e infecção, e casos mais graves da doença que resultaram na Síndrome Respiratória Aguda Grave (SRAG). No Brasil, o registro do primeiro caso ocorreu em fevereiro de 2020 no estado de São Paulo. Atualmente configura-se como uma emergência em saúde pública devido ao seu elevado grau de disseminação e de mortalidade ${ }^{(1)}$.

A doença da COVID-19 é uma doença grave, geralmente apresenta os sintomas de febre, tosse, dispneia, mialgia e/ou fadiga e pode infectar qualquer pessoa em qualquer idade e, em alguns casos pode ser assintomática. Manifesta-se de forma mais grave nos idosos e naqueles com uma doença subjacente, como hipertensão, doença cardiovascular, diabetes mellitus, do Hospital Universitário da Doença renal, doença pulmonar obstrutiva (DPOC). Pode resultar em insuficiência respiratória progressiva contribuindo para o aumento da taxa de mortalidade ${ }^{(2)}$.

No contexto da pandemia, os sistemas de saúde precisaram se readequar a nova necessidade de enfrentamento da COVID-19. Muitos serviços hospitalares suspenderam os procedimentos cirúrgicos eletivos e mantiveram apenas prioridade nos procedimentos de urgência e emergência, em virtude da necessidade de disponibilizar mais leitos aos pacientes com COVID-19. No entanto, a suspensão desses procedimentos eletivos, mas que apresentavam um caráter de urgência resultou no aumento da mortalidade de pacientes que precisavam realizar alguma modalidade cirúrgica ${ }^{(2)}$.

Portanto, no atual contexto da pandemia por SARS-CoV-2 deve ser implementado um protocolo para os pacientes que necessitam realizar um procedimento cirúrgico, definindo como dever ser realizada a preparação pré-operatória, o gerenciamento do período intraoperatório e a vigilância pósoperatória com a finalidade de evitar complicações e garantir a segurança dos pacientes e da equipe de saúde ${ }^{(3)}$.

O retorno das cirurgias eletivas na fase de estabilização da pandemia da COVID-19 demonstrou as dificuldades das instituições de saúde na determinação do tempo ideal para se realizar os procedimentos cirúrgicos em pacientes que se recuperaram da COVID-19, já que a doença pode complicar o andamento perioperatório e elevar a taxa de mortalidade mesmo em cirurgias de pequeno porte ${ }^{(3)}$.

Devido à escassez de informações acerca das características clínicas e dos resultados de pacientes infectados pela COVID-19 submetidos a cirurgias, tendo em vista que pacientes assintomáticos com COVID-19 sofrem rápida deterioração do 
estado clínico após a realização de cirurgias e, por isso, a área cirúrgica, no contexto da pandemia, enfrenta uma necessidade de diversas adaptações. Nesse sentido, foi proposta esta revisão integrativa da literatura com o objetivo de identificar, na literatura científica, as evidências disponíveis em relação às principais complicações póscirúrgicas em pacientes com COVID-19, ampliando o conhecimento e o embasamento científico para a prática assistencial ao indivíduo infectado pelo Coronavíruscom necessidade de submeter-se a um procedimento cirúrgico.

\section{MÉTODOS}

Trata-se de uma revisão integrativa realizada no mês de junho de 2021, seguindo as seguintes etapas: identificação do problema; busca na literatura; avaliação dos dados; análise dos dados e apresentação dos dados.

A estratégia de busca dos estudos primários e questão norteadora seguiram a estratégia PICOS (Population, Intervention, Comparison Group, Outcomes and Study Design) ${ }^{(4)}$.

A população $(\mathrm{P})$ de interesse consistiu em pacientes submetidos a procedimento cirúrgico após infecção por COVID-19; Nesse estudo, a intervenção (I) foi substituída por exposição (E) por se tratar de um evento prejudicial à saúde, portanto, não passível de randomização. A infecção pelo COVID-19 foi considerada a exposição (E). Não houve grupo comparação ou controle (C). O desfecho (outcome - $\mathrm{O}$ ) foi às complicações pós-operatória após COVID-19. E o tipo de estudo (S) foi de estudos originais com síntese qualitativa.

Para a realização da pesquisa foram seguidas seis etapas para condução da presente revisão de literatura: $1^{\mathrm{a}}$ fase Elaboração da pergunta norteadora; $2^{\mathrm{a}}$ fase Busca ou amostragem na literatura; $3^{\mathrm{a}}$ fase Coleta de dados; $4^{\mathrm{a}}$ fase - Análise crítica dos estudos incluídos; $5^{\mathrm{a}}$ fase - Discussão dos resultados; $6^{a}$ fase - Apresentação da revisão integrativa.

A $1^{\text {a }}$ fase "elaboração da pergunta norteadora" pode ser definida como a fase de maior importância na elaboração da revisão sendo: Quais as atuais evidências científicas disponíveis na literatura a respeito das complicações pós-cirurgia em pacientes após infecção por COVID-19?

A $2^{\text {a }}$ fase "Busca ou amostragem na literatura". Para este estudo foram elegidas as seguintes bases de dados eletrônicas, para serem feitas as pesquisas: Medical Literature Analysis and Retrieval System Online (MedLine/PubMed), Biblioteca Virtual em Saúde (BVS), Eletronic Library Online (SciELO), Literatura Latino-Americana (LILACS).

Para conduzir as estratégias de busca com foco em resgatar o maior número de estudos que respondessem à questão norteadora, utilizou-se um vocabulário 
estruturado de Descritores em Ciências da Saúde (DeCS), combinadas com os operadores booleanos AND e OR. A seguinte estratégia de busca foi usada para elencar os estudos primários: "Novo coronavírus" OR "Novel Coronavirus" OR "SARS-CoV-2" OR "COVID-19" AND "Trauma" OR "Emergency trauma care" OR "Atendimento de Emergência ao trauma" AND "Emergency surgery” OR “Cirurgia de emergência” AND "Infection prevention" OR "prevenção de infecção".

Os critérios de elegibilidade foram norteados pela questão da pesquisa. Foram incluídos estudos que abordavam complicações cirúrgicas em pacientes após infecção por COVID-19, publicados em português e inglês, no período de 2019 a 2021, que tivesse os descritores pesquisados. Os estudos excluídos foram estudos secundários, cartas de resposta, editoriais e artigos duplicados. Seguindo os critérios de pesquisa, elaborou-se um diagrama de fluxo (conforme o PRISMA) na pesquisa do banco de dados ${ }^{(5)}$.

A $3^{\text {a }}$ fase foi realizada a coleta de dados extraindo os dados de identificação dos artigos (título, ano de publicação e país) e as características metodológicas do estudo (tipo de estudo, sujeito da pesquisa e principais resultados e conclusões) a partir de uma tabela elaborada pelos autores.

A $4^{\mathrm{a}}$ fase $\operatorname{logo}$ em seguida, após o instrumento ter sido preenchido, foi realizada a análise dos resultados, descrevendo e classificando os dados. Depois, os artigos foram agrupados, de acordo com os principais assuntos abordados.

A $5^{\mathrm{a}}$ fase, "Discussão dos resultados", na qual foram efetuadas análises dos dados obtidos e apresentados em forma de tabela, possibilitando identificar a temática desta pesquisa. A 6 a fase "Foi a Apresentação da revisão integrativa", que se relaciona a uma apresentação de todas as informações pertinentes de forma detalhada e objetiva, com descrição clara e completa para a viabilidade da avaliação crítica dos resultados.

\section{RESULTADOS}

A busca inicial resultou em 767 estudos na base de dados do MedLine/PubMed, 12 na Biblioteca Virtual em Saúde (BVS), 02 na SciELO e 88 na LILAC, totalizando 869 publicações. A seguir executou-se a seleção, considerando-se os estudos potencialmente elegíveis, nesta etapa 849 publicações foram eleitas. Desse total, 804 publicações foram excluídas por não se entrar nos critérios de inclusão. Restaram, então, 65 estudos para leitura dos títulos e resumos, após leitura minuciosa foram excluídos 58 estudos por não responderam à questão de pesquisa, foram selecionados sete estudos ao final para a síntese das evidências, de acordo com o modelo PRISMA (Figura 1). 
Figura 1- Fluxograma de seleção dos artigos incluídos.

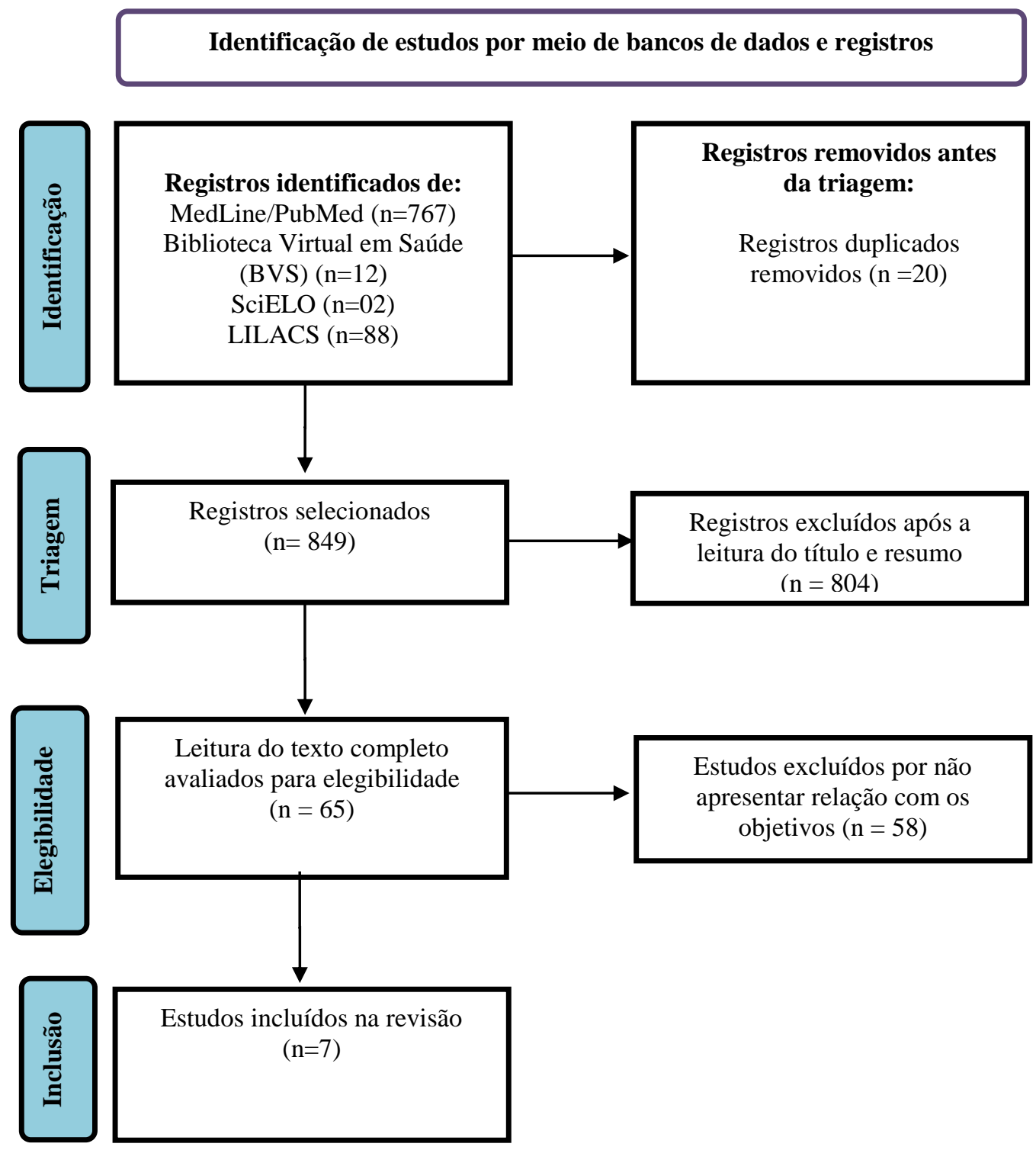

Fonte: http://prisma-statement.org/prismastatement/flowdiagram.aspx.

A análise dos sete artigos que Estados Unidos $(n=1)$, Espanha $(n=1)$, Canadá compuseram a amostra evidenciou que todos $(n=1)$ e um estudo não informou o país de foram oriundos de periódicos internacionais e realização do estudo. publicados na língua inglesa.

A caracterização dos artigos incluídos

Os sete artigos foram realizados de na revisão foi apresentada no Quadro 1. 2020 a 2021, na China/Cingapura ( $\mathrm{n}=3)$, nos 
Quadro 1 - Distribuição das referências incluídas na revisão integrativa, de acordo com ano de publicação, país, autores, tipo de estudo, principais resultados e conclusões.

\begin{tabular}{|c|c|c|c|c|c|}
\hline $\mathbf{N}^{\mathbf{0}}$ & TÍTULO & $\begin{array}{l}\text { ANO E } \\
\text { PAIIS }\end{array}$ & $\begin{array}{l}\text { TIPO DE } \\
\text { ESTUDO }\end{array}$ & $\begin{array}{c}\text { SUJEITO DA } \\
\text { PESQUISA }\end{array}$ & $\begin{array}{c}\text { PRINCIPAIS } \\
\text { RESULTADOS/ } \\
\text { CONCLUSÔES }\end{array}$ \\
\hline 01 & $\begin{array}{l}\text { Emergency trauma } \\
\text { care during the } \\
\text { outbreak of corona } \\
\text { virus disease } 2019 \\
\left.\text { (COVID-19) in }_{\text {China (Yang Li) }}\right)^{(6)} \text {. }\end{array}$ & $\begin{array}{l}2020, \\
\text { Wuhan, } \\
\text { China. }\end{array}$ & $\begin{array}{l}\text { Observacional } \\
\text { descritivo. }\end{array}$ & $\begin{array}{l}\text { Pacientes com } \\
\text { COVID-19 que } \\
\text { necessitaram realizar } \\
\text { procedimento } \\
\text { cirúrgico de } \\
\text { emergência. }\end{array}$ & $\begin{array}{l}\text { Lesão pulmonar } \\
\text { aguda causada por } \\
\text { COVID-19 pode } \\
\text { existir no pré- } \\
\text { operatório ou piorar } \\
\text { no pós-operatório; } \\
\text { Ocorrência de } \\
\text { insuficiência } \\
\text { respiratória e choque } \\
\text { séptico no pós- } \\
\text { operatório. }\end{array}$ \\
\hline 02 & $\begin{array}{l}\text { Perioperative } \\
\text { Considerations in } \\
\text { Urgent Surgical } \\
\text { Care of Suspected } \\
\text { and Confirmed } \\
\text { Coronavirus Disease } \\
\text { 2019 Orthopaedic } \\
\text { Patients: Operating } \\
\text { Room Protocols and } \\
\text { Recommendations } \\
\text { in the Current } \\
\text { Coronavirus Disease } \\
\text { 2019 Pandemic } \\
\text { (Mohamed) }\end{array}$ & $\begin{array}{l}2020, \\
\text { Estados } \\
\text { Unidos. }\end{array}$ & $\begin{array}{l}\text { Descritivo } \\
\text { transversal. }\end{array}$ & $\begin{array}{l}\text { Pacientes ortopédicos } \\
\text { urgentes COVID-19 } \\
\text { confirmados. }\end{array}$ & $\begin{array}{l}\text { Um subgrupo de } \\
\text { COVID-19 grave } \\
\text { pode causar uma } \\
\text { "síndrome de } \\
\text { tempestade de } \\
\text { citocinas", que é uma } \\
\text { síndrome } \\
\text { hiperinflamatória sub- } \\
\text { reconhecida } \\
\text { caracterizada por um } \\
\text { hiperfulminante e } \\
\text { fatal com falência de } \\
\text { múltiplos órgãos. }\end{array}$ \\
\hline 03 & $\begin{array}{l}\text { Emergency Surgery } \\
\text { and Trauma } \\
\text { Care During } \\
\text { COVID-19 } \\
\text { Pandemic. } \\
\text { Recommendations } \\
\text { of the Spanish } \\
\text { Association of } \\
\text { Surgeons (Aranda- } \\
\text { Narváez) }^{(8)} \text {. }\end{array}$ & $\begin{array}{l}\text { 2020, } \\
\text { Espanha }\end{array}$ & $\begin{array}{l}\text { Descritivo } \\
\text { retrospective. }\end{array}$ & $\begin{array}{l}\text { Cirurgia de } \\
\text { emergência e } \\
\text { cuidados ao paciente } \\
\text { politraumatizado. }\end{array}$ & $\begin{array}{l}\text { A própria lesão } \\
\text { cirúrgica poderia ser } \\
\text { um gatilho para uma } \\
\text { resposta inflamatória } \\
\text { imediata } \\
\text { desproporcional que } \\
\text { em pacientes } \\
\text { infectados por } \\
\text { COVID-19 pode ser } \\
\text { deletéria. }\end{array}$ \\
\hline 04 & $\begin{array}{l}\text { The management of } \\
\text { surgical patients in } \\
\text { the emergency } \\
\text { setting during } \\
\text { COVID-19 } \\
\text { pandemic: the } \\
\text { WSES position } \\
\text { paper }{ }^{(9)} \text {. }\end{array}$ & $\begin{array}{l}2021, \\
\text { Não } \\
\text { informado } \\
\text {. }\end{array}$ & $\begin{array}{l}\text { Descritivo } \\
\text { retrospectivo. }\end{array}$ & $\begin{array}{l}\text { Cirurgias de } \\
\text { emergência para } \\
\text { pacientes cirúrgicos }\end{array}$ & $\begin{array}{l}\text { A cirurgia pode ter } \\
\text { um impacto negativo } \\
\text { em pacientes COVID- } \\
19, \text { mesmo se são } \\
\text { assintomáticos, com } \\
\text { mortalidade de } 20,5 \% \\
\text { e a necessidade } \\
\text { potencial de UTI pós- } \\
\text { operatória cuidado. }\end{array}$ \\
\hline
\end{tabular}




\begin{tabular}{|c|l|l|l|l|l|}
\hline $\mathbf{0 5}$ & $\begin{array}{l}\text { Clinical } \\
\text { characteristics and } \\
\text { outcomes of patients } \\
\text { undergoing } \\
\text { surgeries during the } \\
\text { incubation period of } \\
\text { COVID-19 infection } \\
\text { (10) }\end{array}$ & $\begin{array}{l}\text { 2020, } \\
\text { Wuhan, } \\
\text { China. }\end{array}$ & $\begin{array}{l}\text { Descritivo } \\
\text { retrospectivo. }\end{array}$ & $\begin{array}{l}\text { Pacientes submetidos } \\
\text { a cirurgias seletivas } \\
\text { durante o período de } \\
\text { incubação do } \\
\text { COVID-19. }\end{array}$ & $\begin{array}{l}\text { A COVID-19 está } \\
\text { associada a mau } \\
\text { prognóstico para } \\
\text { pacientes submetidos } \\
\text { à cirurgia, } \\
\text { especialmente para } \\
\text { aqueles com doenças } \\
\text { crônicas. }\end{array}$ \\
\hline $\mathbf{0 6}$ & $\begin{array}{l}\text { Mortality and } \\
\text { pulmonary } \\
\text { complications in } \\
\text { patients undergoing } \\
\text { surgery with } \\
\text { perioperative SARS- } \\
\text { CoV-2 infection: an } \\
\text { international cohort } \\
\text { study }\end{array}$ & $\begin{array}{l}\text { (11). } \\
\text { Chingapura }\end{array}$ & $\begin{array}{l}\text { Descritivo } \\
\text { transversal. }\end{array}$ & $\begin{array}{l}\text { Pacientes submetidos } \\
\text { à cirurgia que tiveram } \\
\text { infecção por SARS- } \\
\text { CoV-2 confirmada 7 } \\
\text { dias antes ou 30 dias } \\
\text { após a cirurgia. }\end{array}$ & $\begin{array}{l}\text { O desfecho primário } \\
\text { foi mortalidade, } \\
\text { seguido de } \\
\text { complicações } \\
\text { pulmonares. }\end{array}$ \\
\hline $\begin{array}{l}\text { Postoperative } \\
\text { outcomes in surgical } \\
\text { COVID19 patients: } \\
\text { a multicenter cohort } \\
\text { study }(12)\end{array}$ & $\begin{array}{l}2021, \\
\text { Canadá }\end{array}$ & $\begin{array}{l}\text { Descritivo } \\
\text { longitudinal. }\end{array}$ & $\begin{array}{l}\text { Pacientes COVID-19 } \\
\text { submetidos à cirurgia. }\end{array}$ & $\begin{array}{l}\text { Complicações } \\
\text { pulmonares } \\
\text { Complicações } \\
\text { infecciosas (não } \\
\text { pulmonares) } \\
\text { Lesão renal aguda } \\
\text { Complicações } \\
\text { tromboembólicas }\end{array}$ \\
\hline
\end{tabular}

\section{Fonte: Os autores}

Pela análise do Quadro 1, os sujeitos da pesquisa foram definidos como pacientes com COVID-19 que necessitaram realizar procedimentos cirúrgicos de emergência (6, 9, 12), pacientes ortopédicos urgentes COVID$19 \operatorname{confirmados}^{(7)}$, cirurgia de emergência e cuidados ao paciente politraumatizado ${ }^{(8)}$, pacientes submetidos a cirurgias seletivas durante o período de incubação do COVID-19 (10), pacientes submetidos à cirurgia que tiveram infecção por SARS-CoV-2 confirmada 7 dias antes ou 30 dias após a cirurgia $^{(11)}$.

O procedimento cirúrgico teve um impacto negativo no quadro clínico desses pacientes, mesmo os que estavam assintomáticos no período pré-operatório tiveram um significativo aumento da mortalidade pós-cirúrgica ${ }^{(9)}$. Como principais complicações identificadas no período pósoperatórios desses pacientes citados nos estudos que apresentaram infecção por COVID-19 ativa ou recente foram: lesão pulmonar aguda causada por COVID-19, insuficiência respiratória, choque séptico, "síndrome de tempestade de citocinas", que é uma síndrome hiperinflamatória subreconhecida caracterizada por um hiperfulminante e fatal com falência de múltiplos órgãos, a própria lesão cirúrgica 
como um fator para desencadear uma resposta inflamatória imediata desproporcional, lesão renal aguda, complicações tromboembólicas e o aumento da necessidade de internação em Unidade de Terapia Intensiva (UTI).

\section{DISCUSSÃO}

A pandemia causada pelo COVID-19 tem preocupado as autoridades e os profissionais da saúde por se tratar de um dos maiores desafios enfrentado atualmente nos serviços de saúde, pois pouco se sabe sobre a doença, motivo pelo qual muitas das condutas que já foram realizadas desde o início da doença estavam baseadas no empirismo ${ }^{(13)}$.

Os pacientes cirúrgicos, tanto os que tiveram contato com a COVID-19 antes de submeter-se a um procedimento cirúrgico como os que estão expostos a um risco elevado de contraírem a doença no pósoperatório apresentam um risco elevado de morbimortalidade e uma chance maior de necessitarem de internação em Unidade de Terapia Intensiva ${ }^{(13)}$.

A partir da análise dos resultados verificou-se que a maior parte dos pacientes envolvidos nos estudos apresentava história de exposição prévia ao COVID-19 antes da admissão hospitalar, alguns possuíam sintomas e outros não apresentavam qualquer sinal ou sintoma de COVID-19 antes da cirurgia, no entanto, foram submetidos a procedimento cirúrgico devido a serem situações de emergência ${ }^{(14)}$.

Pacientes com COVID-19 apresentam maior taxa de mortalidade no período pósoperatório, e Os pacientes em pós-operatório com COVID-19 necessitaram de cuidados em Unidades de Terapia Intensiva em uma proporção muito maior do que em pacientes COVID-19 hospitalizados sem cirurgia, sendo que a maioria dos pacientes era mais velha, tinham mais comorbidades subjacentes e tempo cirúrgico mais longo e foi submetido a cirurgias mais difíceis. Isso sugere que a idade avançada associada à comorbidades, tempo cirúrgico e dificuldades cirúrgicas podem ser fatores de risco para resultados desfavoráveis ${ }^{(15)}$.

O trauma e a cirurgia podem prejudicar a função imunológica do paciente. Clinicamente, alguns pacientes assintomáticos com COVID-19 sofrem rápida deterioração após a cirurgia. Cirurgiões e anestesiologistas devem estar cientes de que lesão pulmonar aguda causada por COVID-19 pode existir no pré-operatório ou piorar no pós-operatório ${ }^{(16)}$.

É oportuno destacar que os pacientes submetidos a procedimentos cirúrgicos com infecção prévia por COVID-19 foram identificados complicações pulmonares pósoperatórias em metade dos pacientes com infecção prévia por COVID-19 ${ }^{(17)}$.

As complicações pulmonares foram definidas como pneumonia, síndrome do 
desconforto respiratório agudo (SDRA) ou ventilação pós-operatória inesperada. Estas são as complicações pulmonares mais frequentes relacionadas ao COVID-19 em pacientes cirúrgicos. Desfechos secundários adicionais incluíram embolia pulmonar, internação em UTI, reoperação, mortalidade em 7 dias e tempo de internação ${ }^{(18)}$.

Portanto, a caracterização e diferenciação de sintomas podem ajudar a equipe de saúde a identificar pacientes com potenciais desfechos não favoráveis. Assim, a avaliação pós-operatória objetiva identificar possíveis complicações decorrentes do quadro clínico do paciente ${ }^{(19)}$.

Cada instituição de saúde e equipe cirúrgica deve revisar cuidadosamente todos os procedimentos eletivos com o objetivo avaliar o custo benefício do procedimento em meio ao risco de prováveis complicações em decorrência da COVID-19, prevenindo os eventos adversos nesses pacientes de baixa morbimortalidade, mas que podem cursar como procedimentos fatais em infectados pelo COVID-19 ${ }^{(20)}$.

Portanto, em virtude do contexto atual, deve-se adiar ou cancelar cirurgias eletivas não essenciais e avaliar criteriosamente o momento da execução e os riscos e benefícios na realização desses procedimentos cirúrgicos eletivos essenciais que possam ter repercussões clínicas importantes pela não realização cirúrgica ${ }^{(21)}$.
Salienta-se que a avaliação epidemiológica local e regional é um fator determinante e fundamental de ser avaliado antes de considerar a retomada da realização de cirurgias eletivas, recomenda-se como momento quando ocorrer à redução sustentada de novos casos da COVID-19 no mínimo de 14 dias consecutivos na área geográfica $^{(15)}$.

As instituições de saúde devem formular estratégias e diretrizes de qualidade e segurança cirúrgica voltada para o atendimento desses pacientes, além da avaliação das condições estruturais do serviço de saúde, disponibilidade de um quantitativo de leitos hospitalares para atender aos pacientes pós-cirúrgicos além da demanda dos casos de COVID-19 ${ }^{(21)}$.

Para auxiliar os serviços de saúde, a Agência Nacional de Vigilância Sanitária (ANVISA) lançou uma nota técnica com orientações para a prevenção e o controle das infecções pelo novo coronavírus (SARSCoV-2) em procedimentos cirúrgicos a fim de garantir a retomada segura dos procedimentos cirúrgicos eletivos, definindo como deve ser feita a programação cirúrgica, a composição da equipe cirúrgica e as medidas de segurança adotadas no ambiente cirúrgico ${ }^{(15)}$.

\section{CONCLUSÃO}

A cirurgia tem um impacto negativo em pacientes com COVID-19, mesmo os que 
são assintomáticos, elevando o risco de mortalidade e a necessidade potencial de internação pós-operatória em Unidade de Terapia Intensiva (UTI).

As pesquisas que integraram a presente revisão revelam que as principais complicações pós-operatórias em pacientes infectados por COVID-19 ou que estavam recuperados de uma infecção recente da doença são: as complicações pulmonares, complicações infecciosas (não pulmonares), lesão renal aguda, complicações tromboembólicas, choque séptico e a síndrome de tempestade de citocinas, caracterizada como uma síndrome hiperinflamatória sub-reconhecida resultando em falência de múltiplos órgãos por seu estado hiperfulminante.

Diante dos resultados encontrados, constata-se que todos os procedimentos não urgentes devem ser adiados até que o paciente tenha cumprido com os critérios de isolamento e cuidados de transmissão e a doença tenha entrado na fase de recuperação.

Acredita-se que o presente estudo seja capaz de incentivar novas produções científicas relacionadas ao tema, tendo em vista que é um problema atual e de grande impacto para a saúde pública, representando um potencial agente de complicações para os pacientes cirúrgicos.

Como limitação na realização do estudo, destacamos o número reduzido, tanto em literaturas nacionais quanto internacionais sobre a temática. Portanto, verifica-se a necessidade de pesquisas futuras sobre o tema, a fim de contribuir para o esclarecimento sobre as complicações ainda desconhecidas da COVID-19 em pacientes submetidos à cirurgia.

\section{REFERÊNCIAS}

1. Brito SBP, Braga I O, Cunha CC, Palácio MAV, Takenami I. Pandemia da COVID-19: o maior desafio do século XXI COVID-19. Vigil. sanit. debate 2020;8(2):54-63. [acesso em 06 jun 2021]; Disponível em: https://visaemdebate.incqs.fiocruz.br/i ndex.php/visaemdebate/article/view/1 $\underline{531 / 1148}$

2. Nahshon C, Bitterman A, Haddad R, Hazzan D, Lavie O. Hazardous Postoperative Outcomes of Unexpected COVID-19 Infected Patients: A Call for Global Consideration of Sampling all Asymptomatic Patients Before Surgical Treatment. World J Surg. V.16, p.1-5, 2020. [acesso em 07 jun 2021]; Disponível em: https://europepmc.org/backend/ptpmcr ender.fcgi?accid=PMC7229873\&blobt ype $=$ pdf

3. Marques LC, Lucca DC, Alves, EO, Fernandes, GCM, Nascimento KC. COVID-19: Cuidados de enfermagem para segurança no atendimento de serviço pré-hospitalar móvel. Texto \& Contexto Enfermagem 2020, v. 29: e20200119. [acesso em 07 jun 2021]; Disponível em: https://www.scielo.br/j/tce/a/TsWF5L WQStRtzYJCnP9jvvK/?lang=pt\&for $\underline{\text { mat }=p d f}$ 
4. Methley AM, Campbell S, ChewGraham C, Mcnally R, Cheraghi-Sohi S. PICO, PICOS and SPIDER: a comparison study of specificity and sensitivity in three search tools for qualitative systematic reviews. BMC Health Services Research (2014). [acesso em 10 jun 2021]; Disponível em:

https://bmchealthservres.biomedcentra 1.com/track/pdf/10.1186/s12913-0140579-0.

5. Moher D, Liberati A, Tetzlaff J, Altman DG; PRISMA Group. Preferred reporting items for systematic reviews and meta-analyses: the PRISMA statement. Int J Surg. 2010; 8(5):336-41. doi: 10.1016/j.ijsu.2010.02.007. Epub 2010 Feb 18. Erratum in: Int J Surg. 2010; 8(8):658. PMID: 20171303. [acesso em 10 jun 2021]; Disponível em:

https://reader.elsevier.com/reader/sd/p ii/S1743919110000403?token $=31548$ CA75EF5E

6. Li Y, Zeng L, Li Z, Mao Q, Liu D, Zhang L, et al. Emergency trauma care during the outbreak of corona virus disease 2019 (COVID-19) in China. World Journal of Emergency Surgery (2020). [acesso em 23 jun 2021]; Disponível em: https://wjes.biomedcentral.com/article s/10.1186/s13017-020-00312-5

7. Awad ME, Rumley JCL, Vazquez JA, Devine JG. Perioperative Considerations in Urgent Surgical Care of Suspected and Confirmed COVID-19 Orthopaedic Patients: Operating Room Protocols and Recommendations in the Current COVID-19 Pandemic. J Am Acad Orthop Surg. 2020; 28(11): 451-463. doi:10.5435/JAAOS-D-20-00227 [acesso em 23 jun 2021]; Disponível em:

https://www.ncbi.nlm.nih.gov/pmc/art icles/PMC7197335/

8. Aranda-Narváez JM, Tallón-Aguilar L, Pareja-Ciuró $\quad$ F, Martín-Martín G, González-Sánchez AJ, Rey-Simó I, et al. Emergency Surgery and Trauma Care During COVID-19 Pandemic. Recommendations of the Spanish Association of Surgeons. cirugía española . 2020; [acesso em 24 jun 2021]; Disponível em: https://pubmed.ncbi.nlm.nih.gov/3243 $\underline{9139 /}$

9. Simone BD, Chouillard E, Sartelli M, Biffl WL, Saverio SD, Moore EE, et al. The management of surgical patients in the emergency setting during COVID-19 pandemic: the WSES position paper. [acesso em 24 jun 2021]; Disponível em: https://www.scienceopen.com/docume $\underline{\text { nt? }}$ vid $=6414 \mathrm{e} 1 \mathrm{~b} 0-99 \mathrm{fd}-41 \mathrm{~d} 7-905 \mathrm{a}-$ ccce0c81f621.

10. Lei S, Jiangb F, Sua W, Chen C, Jingli Chen, Mei W, et al. Clinical characteristics and outcomes of patients undergoing surgeries during the incubation period of COVID-19 infection. EClinicalMedicine 21 (2020) 100331. [acesso em 25 jun 2021]; Disponível em: https://www.thelancet.com/journals/ec linm/article/PIIS2589-5370(20)300754/fulltext.

11. COVIDSurg Collaborative. Mortality and pulmonary complications in patients undergoing surgery with perioperative SARS-CoV-2 infection: an international cohort study. The Lancet.com Vol 396 July 4, 2020. [acesso em 25 jun 2021]; Disponível em:

https://www.thelancet.com/journals/la ncet/article/PIIS0140-6736(20)31182$\underline{\mathrm{X} / \text { fulltext }}$ 
12. Carrier FM, Amzallag E, Lecluyse V, Côté G, Couture EJ, D'Aragon F, et al. Postoperative outcomes in surgical COVID19 patients: a multicenter cohort study. Carrier et al. BMC Anesthesiology (2021) 21:15. [acesso em 26 jun 2021]; Disponível em: https://bmcanesthesiol.biomedcentral. com/articles/10.1186/s12871-02101233-9

13. Nunes VRT, Dias JSR, Nogueira HPP, Assis AC, Brenck LF, Andrade GL, Aguiar-Jr MC. Cuidados cirúrgicos durante a pandemia mundial do COVID-19: rotinas alternativas para minimizar os riscos. Rev Med Minas Gerais 2021; 31: e-31701. [acesso em 07 jul 2021]; Disponível em: http://www.rmmg.org/artigo/detalhes/ 2774

14. Wang D, Hu B, Hu C, Zhu F, Liu X, Zhang $\mathrm{J}$ et al. Clinical characteristics of 138 hospitalized patients with 2019 novel coronavirus-infected pneumonia in Wuhan, China. JAMA 2020. [acesso em 07 jul 2021]; Disponível em:

https://jamanetwork.com/journals/jam a/fullarticle/2761044.

15. Nota técnica GVIMS/GGTES/ANVISA n ${ }^{\circ}$ 06/2020 Orientações para a prevenção e o controle das infecções pelo novo coronavírus (sars-cov-2) em procedimentos cirúrgicos - Revisão: 30/03/2021 (Complementar à nota técnica GVIMS/GGTES/ANVISA N ${ }^{\circ}$ 04/2020). [recurso eletrônico]; [acesso em 08 jul 2021]; Disponível em: https://ameci.org.br/wpcontent/uploads/2021/04/NOTATECNICA-06_2020-CIRURGIAS30.03.2021-para-o-site.pdf

16. Hojaij FC, Chinelatto LA, Boog GHP, Kasmirski JA, Lopes JVZ, Sacramento FM. Surgical Practice in the Current COVID-19 Pandemic: A Rapid Systematic Review. Clinics (São Paulo), v.75, p. e1923, 2020. [acesso em 09 jul 2021]; Disponível em:

https://www.scielo.br/j/clin/a/BqHnTft $\underline{\text { SkJcqCZ4zJZ8RZdP/?lang=en }}$

17. Jinpeng Li, Rongfen Gao, Gaosong $\mathrm{Wu}$, Xiaolin $\mathrm{Wu}$, Zeming Liu, Hongjing Wang, Yihui Huang, Zhenyu Pan, Jincao Chen, Xiaohui $\mathrm{Wu}$. Clinical characteristics of emergency surgery patients infected with coronavirus disease 2019 (COVID-19) pneumonia in Wuhan, China [acesso em: 09 jul 2021]. Disponível em: https://www.sciencedirect.com/scienc e/article/pii/S0039606020302865.

18. Aminian A, Safari S, RazeghianJahromi A, Ghorbani M, Delaney CP. COVID-19 Outbreak and Surgical Practice: Unexpected Fatality in Perioperative Period. Ann Surg. 2020 Mar 26. [acesso em: 09 jul 2021]. Disponível em: https://www.ncbi.nlm.nih.gov/pmc/art icles/PMC7188030/pdf/ansu-publishahead-of-print10.1097.sla.0000000000003925.pdf

19. Coccolini F, Perrone G, Chiarugi M, Di Marzo F, Ansaloni L, Scandroglio I, et al. Surgery in COVID-19 patients: operational directives. World J Emerg Surg. V.15, n.25, 2020. [acesso em: 09 jul 2021]. Disponível em: https://www.ncbi.nlm.nih.gov/pmc/art icles/PMC7137852/.

20. Pereira X, Lima D1, Moran-Atkin E, Malcher F. Para onde foram os pacientes cirúrgicos durante a pandemia de COVID-19? Rev Col Bras Cir 47: e20202733. [acesso em: 12 jul 2021]. Disponível em: https://www.scielo.br/j/rcbc/a/NdcQdj 
mT9HwVqmzC8vdkX9w/?format=pd f\&lang=pt.

21. Nunes VRT, Dias JSR, Nogueira HPP, Assis AC, Brenck LF, Andrade GL, Aguiar-Jr MC. Cuidados cirúrgicos durante a pandemia mundial do COVID-19: rotinas alternativas para minimizar os riscos. Rev Med Minas Gerais 2021; 31: e-31701. [acesso em: 12 jul 2021]. Disponível em: http://www.rmmg.org/artigo/detalhes/ $\underline{2774}$

Autor correspondente

Aline Sousa Falcão, Rua Z, quadra 01, casa 07 Planalto Anil III, CEP: 65050879. Telefone: +55(98) 98784-5286, e-mail: alinesousafalcao19@gmail.com alinefalcao.rims@huufma.br

Submissão: 2021-09-06

Aprovado: 2021-10-28 